\title{
On Temporality in Discourse Annotation: Theoretical and Practical Considerations
}

\author{
Jacqueline Evers-Vermeul \\ Utrecht Institute of Linguistics OTS, Utrecht University \\ Trans 10, 3512 JK, Utrecht, The Netherlands
}

J.EVERS@UU.NL

Jet Hoek

Utrecht Institute of Linguistics OTS, Utrecht University

Trans 10, 3512 JK, Utrecht, The Netherlands

\author{
Merel C.J. Scholman \\ Language Science and Technology, Saarland University \\ Campus C7.4, 66123, Saarbrücken, Germany
}

M.C.J.SCHOLMAN@COLI.UNI-SAARLAND.DE

Editor: Maite Taboada

Submitted 11/2016; Accepted 03/2017; Published online 05/2017

\begin{abstract}
Temporal information is one of the prominent features that determine the coherence in a discourse. That is why we need an adequate way to deal with this type of information during discourse annotation. In this paper, we will argue that temporal order is a relational rather than a segmentspecific property, and that it is a cognitively plausible notion: temporal order is expressed in the system of linguistic markers and is relevant in both acquisition and language processing. This means that temporal relations meet the requirements set by the Cognitive approach of Coherence Relations (CCR) to be considered coherence relations, and that CCR would need a way to distinguish temporal relations within its annotation system. We will present merits and drawbacks of different options of reaching this objective and argue in favor of adding temporal order as a new dimension to CCR.

Keywords: coherence relations, Cognitive approach to Coherence relations (CCR), discourse
\end{abstract} annotation, discourse connectives, language acquisition, language processing, temporality

\section{Introduction}

One of the prominent features that determine the coherence of a discourse is temporality. Zwaan (1996: 1196) has noticed this for temporality at the sentence level in narrative types of discourse: "Temporal information is ubiquitous to narratives. Not only does every sentence in a narrative carry temporal information, temporal information can be expressed in every major word class." Temporality is also highly relevant at the discourse level, where the states and events reported in individual clauses are ordered relative to each other. For instance, in examples (1)-(3), the 
temporal ordering of the segments is the most distinctive feature of the relation between the first $\left(\mathrm{S}_{1}\right)$ and the second clause $\left(\mathrm{S}_{2}\right)$.

(1) [I visited my grandmother $] \mathrm{S}_{1}$ before [I went shopping.] $\mathrm{S}_{2}$

(2) [I went shopping $] \mathrm{S}_{1}$ after [I visited my grandmother.] $\mathrm{S}_{2}$

(3) [I visited my grandmother.] $\mathrm{S}_{1}$ Then [I went shopping. $] \mathrm{S}_{2}$

Considering that temporal information is so important in a discourse, we need an adequate way to deal with this type of information during annotation efforts at the discourse level. However, there is currently no consensus in the way in which annotation frameworks deal with temporality at the discourse level. We see at least three different approaches in the classification of discourse relations. In the first approach, temporal relations are simply listed among the inventory of discourse relations (e.g., Bunt \& Prasad, 2016; Kehler, 2002). For example, according to Segmented Discourse Representation Theory (SDRT; Asher \& Lascarides, 2003; Reese et al., 2007), examples (1) and (3) constitute a NARRATION relation, whereas (2) would be a PRECONDITION relation.

In the second approach, different types of temporal relations together constitute a separate class of discourse relations (e.g., Halliday \& Hasan, 1976; Hobbs, 1983; Hovy \& Maier, 1995; Longacre, 1983; Martin, 1992). For example, both the Penn Discourse Treebank (PDTB; Prasad et al., 2008) and the Rhetorical Structure Theory Discourse Treebank (RST-DT; Carlson et al., 2003) distinguish a class of TEMPORALS. In the PDTB, this class includes PRECEDENCE relations such as (1) and (3), and SUCCESSION relations such as (2). RST-DT would also classify these relations as belonging to the class of TEMPORALS, but would label (1) TEMPORAL-BEFORE, (2) TEMPORAL-AFTER, and (3) SEQUENCE (see Sanders et al., submitted for details on the exact mapping of different labels across annotation frameworks).

By contrast, the third approach considers temporal order not as a relational, but as a segmentspecific feature expressed by the propositional content of clauses in a text (e.g., Partee, 1984; Hinrichs, 1986; Nerbonne, 1986; Webber, 1988). This view can be found in the Cognitive approach to Coherence Relations (CCR; Sanders, Spooren \& Noordman, 1992, 1993), which does not have a separate category of temporal relations. Sanders et al. (1992) do not claim that temporal order is not relevant to discourse; instead, they argue that temporal relations belong to the class of ADDITIVE relations because "the properties distinguishing temporal relations from other additive relations concern the referential meaning of the individual segments" (1992: 28).

The discrepancy between approaches to temporality raises the following research question: what should be the status of temporality in discourse annotation? Should temporal order be considered a relational feature or a segment-specific feature? This question is also addressed in Kehler (1994) and relates to the definition of a coherence relation. Sanders et al. (1992) define a coherence relation as "an aspect of meaning of two or more discourse segments that cannot be described in terms of the meanings of the segments in isolation. In other words, it is because of this coherence relation that the meaning of the discourse segments is more than the sum of its parts" (Sanders et al., 1992: 2). If temporal ordering is always explicitly encoded in the discourse segments of a text, it should not be considered a relational feature, since temporal ordering would not be part of the relational surplus. In that case, discourse relation inventories should not include temporal relations. However, in Section 2 we will argue, similar to the first and second approach presented above, that information on the temporal ordering of segments in a discourse cannot be seen as merely segment-specific information, and that it should thus be considered a relational feature.

In order for any feature to be considered a relational feature in CCR, the feature needs to be cognitively plausible; that is, it should affect language acquisition and discourse processing, and have unique linguistic markers. This means that in CCR, temporal order is only a relational feature if temporal relations have a special cognitive status that is distinct from other types of 
relations, such as additive and causal relations. In Section 3, we will therefore review empirical evidence supporting the claim that temporal order is indeed cognitively plausible.

If temporal order is indeed a distinct cognitively plausible notion, it would need to be implemented in CCR. Because CCR works with a highly restricted set of annotation dimensions that are relevant for describing all types of coherence relations (Sanders et al., 1992, 1993), it cannot do with a first and straightforward option: to simply acknowledge temporal relation labels in its inventory of end labels, an approach that would be similar to the second approach presented above. In Section 4, we will therefore discuss the merits and drawbacks of several ways in which temporal relations can be included in CCR, and argue in favor of making temporal order an additional dimension. The paper ends with a discussion and conclusion (Section 5).

\section{Temporality: propositional or relational feature?}

Sanders, Spooren and Noordman (1992) are clear proponents of the position that the temporal meaning aspect of coherence relations is a propositional rather than a relational feature. They claim, much like Partee (1984), Hinrichs (1986), Nerbonne (1986), and Webber (1988), "that temporal relations belong to the class of additive relations and that the properties distinguishing temporal relations from other additive relations concern the referential meaning of the individual segments" (Sanders et al., 1992: 28). According to them, "the temporal meaning aspect is to a large degree determined by the referential content of the segments, more than, for instance, the causal meaning aspect" (Sanders et al., 1992: 28).

There do indeed appear to be many segment-specific features that can be addressed under the umbrella term 'temporality'. For example, Adam's (2008) overview of the many dimensions of temporality, in her terms timescapes, contains features such as tempo (speed, pace, rate of change), duration (extent, instantaneity) and temporal modality (past, present, future), all of which are expressed within segments. Likewise, Berman and Slobin's (1994: 19) definition of temporality includes two segment-specific properties: the expression of the location of events on the timeline (past, present and future), and the temporal constituency of events (perfective, imperfective, progressive, iterative; cf. also Comrie, 1976). In this paper, we restrict our discussion of temporality to the third subtype of temporality listed in Berman and Slobin (1994): the temporal relations between events (anteriority, simultaneity and posteriority). This sequential order of the propositions expressed by discourse segments at least has the potential of being a relational and not just a propositional feature. In the remainder of this section, we will look at a variety of segment-specific temporality markers, and show that the presence of such markers is neither necessary nor sufficient for expressing the third subtype of temporality, temporal order.

An example of a relation in which the sequential order of the segments is explicitly encoded in one of the segments is (4). Five minutes later in $\mathrm{S}_{2}$ indicates that $\mathrm{S}_{2}$ took place after $\mathrm{S}_{1}$, and can therefore be considered a sequence marker, which places an event in relation to one or several other events mentioned in the discourse (Bestgen \& Costermans, 1994; Costermans \& Bestgen, 1991).

(4) [Mona received a phone call saying that she had lost her most important client. $]_{\mathrm{s} 1}[$ Five minutes later, she was fired. $]_{\mathrm{s} 2}$

In (4), the temporal progression does not seem to be part of the relational surplus, but rather expressed by the propositional content of $S_{2}$. Note, however, that the presence of such a marker of temporal progression may not be sufficient for making the coherence relation temporal. It is for instance also very reasonable to infer a causal relation between the segments in (4); losing an important client is a plausible reason to be fired.

(5) also contains lexical indicators of time: the prepositional phrases at ten o'clock in $\mathrm{S}_{1}$, and at twelve o'clock in $\mathrm{S}_{2}$. In terms of Costermans and Bestgen (1991; Bestgen \& Costermans, 
1994), both these prepositional phrases serve as anchorage markers, elements that tie segments to an absolute time scale external to the narrative. The temporal progression appears, at first glance, to be entirely signaled by the propositional content of the segments and it seems as if there is no temporal meaning surplus at the relational level.

(5) $[\text { At ten o'clock we had coffee. }]_{\mathrm{S} 1}[\text { At twelve o'clock we had lunch. }]_{\mathrm{S} 2}$

However, even though the absolute time points are given within the segments, the temporal progression between the segments is actually inferred by establishing that the time points are two hours apart. The temporal ordering is therefore due to the fact that the two segments are related to each other; in other words, it is part of the relational surplus. Note also that even in the presence of two anchorage markers a relation need not be temporal. The relation in (5) could also be judged as predominantly comparative or even contrastive.

In addition to lexical items expressing time points, grammatical tense can provide information on the ordering of events mentioned in the discourse (cf. Kehler, 1994). For example, the pluperfect in (6) indicates that the event in $S_{2}$ precedes the event in $S_{1}$ (example taken from Lascarides \& Asher, 1993: 472).

(6) [Max slipped. $]_{\mathrm{S} 1}[\mathrm{He} \text { had spilt a bucket of water. }]_{\mathrm{S} 2}$

Again, it may seem as if there is no temporal meaning surplus at the relational level. However, the pluperfect in itself does not signal anti-chronological order. The reversed temporal order is inferable because of the combination of the two tenses; because the event in $S_{2}$ is in pluperfect tense, it has to be interpreted as having occurred before the event in perfect tense in $S_{1}$. Similarly, the future tense of $S_{1}$ in (7) has to be interpreted as occurring after the perfect tense of $S_{2}$, but this is again due to the combination of tenses between the two clauses. Had $S_{2}$ in (7) also been in future tense, as in (8), the temporal ordering would not have been anti-chronological, but rather chronological or simultaneous, depending on the context.

(7) $[\text { Jane will slip. }]_{\mathrm{S} 1}[\text { Max has spilt a bucket of water. }]_{\mathrm{S} 2}$

(8) [Jane will slip. $]_{\mathrm{S} 1}[\text { Max will help her get up. }]_{\mathrm{S} 2}$

The sequential interpretation of segments also differs depending on whether the segments denote states or events. This distinction includes, but is not restricted to grammatical aspect, as verb semantics can also determine whether something is a state or an event. Event-denoting clauses in past tense, such as $S_{2}$ in (9), tend to be temporally interpreted in sequence, that is, they either precede or follow some other event in discourse. In contrast, state-denoting clauses like $\mathrm{S}_{2}$ in (10) tend to temporally overlap with previous discourse events (Gennari, 2004: 878, example (10) taken from Webber, 1987).

(9) [I went to a party last night. $]_{\mathrm{S} 1}[\mathrm{I} \text { ran into an old friend outside. }]_{\mathrm{S} 2}$

(10) [I went to a party last night. $]_{\mathrm{S} 1}[\text { The music was wonderful. }]_{\mathrm{S} 2}$

If the event-state distinction helps distinguish between temporal sequence and temporal overlap, it may seem as if there is no temporal meaning surplus at the relational level. Again, however, it is the combination of the two clauses and their status as states or events that eventually determines the inferred temporal ordering. This entails that the relevance of the event-state distinction for temporality is located in the relational surplus.

Tense and aspect markers, as well as anchorage markers that signal an absolute time point, and sequential markers that signal the relative time between the segments help us establish the 
temporal order of events in a discourse. As mentioned above, the presence of temporal information within the segments is not sufficient to establish a temporal coherence relation. It is not the mere presence of these signals, but rather the way in which these signals are combined that influences the temporal ordering of adjacent clauses.

In addition, the presence of temporal information within the segments is not a necessary characteristic of temporal relations. The segments of the relations in (11)-(13), for instance, have a highly similar structure, but the specific type of temporal relation differs between the sentences.

(11) [Paul read the paper $]_{\mathrm{S} 1}$ before [going to work. $]_{\mathrm{S} 2}$

(12) [Zoe went to the gym $]_{\mathrm{S} 1}$ after [finishing her exam. $]_{\mathrm{S} 2}$

(13) [Arnold organized his desk $]_{\mathrm{S} 1}$ while [talking to his mother on the phone. $]_{\mathrm{S} 2}$

Examples (11)-(13) illustrate that temporal ordering may not always become apparent from the segments alone. The segments in (11)-(13) are structurally identical, with a simple past in $\mathrm{S}_{1}$ and a gerund in $S_{2}$. Still, the order of the events in (11) is opposite from the order of the events in (12), and in (13), the two segments overlap in time. These orders are indicated by the connectives before, after and while, respectively, linguistic elements that are considered not to be part of the propositional content of a text and that are therefore not part of the discourse segments (cf. Blakemore, 2002; Hall, 2007). Given the absence of anchorage markers or other indicators of time, we cannot represent the differences between (11), (12), and (13) without attributing a temporal meaning relation between the two clauses of each fragment.

It should be noted that for other types of coherence relations, the presence of propositional elements hinting at specific relation types also does not necessarily eliminate the option that what they signal is included in the relational surplus of a coherence relation. For example, the presence of negative elements in $\mathrm{S}_{1}$ or of semantic opposites in both segments hint at a contrastive relation (Asr \& Demberg, 2015), as in (14). Similarly, modal elements such as obviously in (15) hint at a subjective relation (Scheibman, 2002; Traugott, 2010). As with temporal relations, the presence of these propositional elements, however, is neither a required nor sufficient property of the relation at hand. For example, (15) remains a subjective argument-claim relation, even in the absence of obviously.

(14) [Harry never lifted a finger, $]_{\mathrm{s} 1}$ but $[\text { Kyle worked really hard. }]_{\mathrm{S} 2}$

(15) [Jenny obviously went to the party, $]_{\mathrm{S} 1}$ since [she came home at 3 A.M. $]_{\mathrm{S} 2}$

In conclusion, temporal ordering of segments in a discourse cannot be seen as merely segment-specific information. It should thus be seen as a relational feature, even if linguistic indicators of temporality are present within a clause. In other words: temporal relations constitute a specific subset of coherence relations, just like, for instance, causal, conditional and contrastive relations.

\section{Cognitive plausibility of temporality}

In CCR, coherence relations are considered to be cognitive entities because they account for the coherence in the reader's cognitive text representation (Sanders et al., 1992). Working from this assumption, it is expected that the nature of a relation affects discourse processing. In other words, different types of relations, such as causal and additive relations, should require different processing costs and result in different mental representations (Sanders \& Noordman, 2000). This means that if temporal order is to be considered a relational feature rather than a propositional one, the defining features that separate temporal relations from other types of relations should be cognitively plausible. In this section, we will examine three types of evidence to determine whether temporal relations are distinct cognitive entities: a) evidence from the system of 
linguistic markers; b) evidence from language acquisition; and c) evidence from language processing.

\subsection{Linguistic markers of temporality}

Connectives and other relational cue phrases signal certain types of relations; for example, because is used to express causal relations and nevertheless is used to express concessive relations. Readers can use these connectives as so-called processing instructions that signal how the incoming information should be related to the previous discourse segment (Britton, 1994; Canestrelli, Mak \& Sanders, 2013). The category of connectives tends to consist of simple linguistic expressions and idiom chunks (Knott \& Dale, 1994: 45), and is often restricted to grammaticalized linguistic elements that express a two-place semantic relation, have propositional arguments, are not integrated in the predicative structure, and do not vary regarding inflection (see among others Mendes \& Lejeune, 2016; Stede, 2002; but for a more liberal point of view see Prasad, Joshi \& Webber, 2010; Rysová \& Rysová, 2015).

Knott and Dale (1994) have used the existence of such connectives and relational cue phrases to motivate a set of coherence relations. They work from the assumption that "[if] people actually use a particular set of relations when constructing and interpreting text, it is likely that the language they speak contains the resources to signal those particular relations explicitly" (Knott \& Dale, 1994: 44). Similarly, Stukker and Sanders (2012) work from what they call the linguistic categorization hypothesis, adopting a basic assumption of cognitive linguistics that a direct link holds between linguistic categories and cognitive categories, and applying it in a cross-linguistic corpus-based study on different types of causal relations. They assume that "when language users systematically prefer one highly grammaticalized linguistic item over another (even highly similar) one to express a certain type of causal relationship, they assign the relation expressed to a specific conceptual type of causality" (Stukker \&Sanders, 2012: 170). Such an approach thus assumes that "linguistic choices provide a window on speakers' cognitive categorizations" (Stukker \& Sanders, 2012: 170). This assumption can be taken to hold for temporal order as well: if there are frequently used, grammaticalized linguistic markers that distinguish different types of temporal relations, one can assume that temporal order is indeed a relational feature.

In order to determine whether a specific feature, such as temporality, constitutes a type of coherence relation, Knott and Dale (1994) look for relational cue phrases that mark this feature. Using a test for identifying relational cue phrases they gather a corpus of around 200 cue phrases for all kinds of relations. This corpus provides ample evidence for connectives and cue phrases in the temporal domain, such as before, after, as, while, when, and as soon as (Knott and Dale, 1994: 60). Assuming that features need to be cognitively plausible in order to be considered a type of relations, Knott and Dale's (1994) study provides evidence that temporality indeed constitutes a separate class of relations.

At first glance, two frequent phenomena in the use of linguistic markers seem to provide evidence to counter this conclusion: 1) underspecification, the fact that connectives and relational cue phrases may feature in a more specific type of relation that they do not encode themselves; and 2) polysemy, the fact that connectives may encode different types of coherence relations. We will discuss these phenomena in turn.

Consider the underspecified relations in (16) and (17) (the latter taken from Mak \& Sanders, 2013: 1415):

(16) Mary burst into tears after her boss fired her.

(17) The boy quarreled with his father when he did not get permission to go out.

In (16) and (17), "the semantics of the connective that is used to indicate the link does not fully match the semantics of the relation" (Spooren, 1997: 150). The two segments present events that 
do not only have a temporal order, but plausibly also a causal relation: Mary likely cried because she got fired, and the boy likely quarreled with his father because he did not get permission to go out. As these paraphrases illustrate, underspecified relations can be recognized by exchanging the underspecified connective for a more specific counterpart (cf. also Givón, 1990: 828). In an eyetracking experiment, Mak and Sanders (2013) have shown that, triggered by the implicit causality verb quarreled in $S_{1}$, readers indeed arrived at a causal interpretation of (17) and similar sentences, even in the presence of the temporal marker when. This resulted in faster reading times throughout $\mathrm{S}_{2}$, compared to sentences without a causal interpretation.

One could argue that because the temporal connectives in (16) and (17) can be used in other types of relations, they cannot be taken as evidence that temporal order is a cognitively plausible feature. However, it should be noted that non-temporal connectives can feature in underspecified relations as well: for example, and can be used to mark additive, contrastive, temporal, and causal relations, and but can be used to mark both contrastive (or negative additive) and concessive (or negative causal) relations. Language users arrive at a richer interpretation than the connective actually encodes due to a highly common process referred to as pragmatic enrichment or conversational implicature (Grice, 1989; Levinson, 2000). Still, the fact remains that connectives such as after and when do occur in relations in which a causal implicature is less likely or not possible at all. The fact that these connectives actually encode temporal ordering, and therefore provide temporal processing instructions to the reader, is still in line with the original linguistic categorization hypothesis.

Another frequent linguistic phenomenon that seems to serve as a counter-argument is polysemy; certain connectives encode not only temporality, but also some other type of relation. For example, in (18), the relation is temporal as well as contrastive: the bad performance of the soccer team is contrasted with the relatively good performance four years earlier. In (19), the contrastive interpretation of while is even the only one available in a context where Mary's father has deceased.

(18) The Dutch soccer team failed to qualify for the 2016 European Cup, while two years before the team had reached the semi-finals of the World Cup.

(19) Mary likes oysters, while her father Bill hated them.

Again, pragmatic enrichment is involved, but this time in the diachronic development of the connective at hand. Traugott (1995) illustrates how elements from the pragmatic context in which an expression typically occurs can become part of a word's conventional meaning, a process referred to as "the semanticization of pragmatics" (see Barlow \& Kemmer, 2000; Traugott, 2011). Middle English while 'during', which was purely temporal, has developed into Modern English while 'although', because the pragmatic inference of "surprise concerning the overlap in time or the relations between event and ground" eventually became conventionalized in the connective (Traugott, 1995: 41; cf. also González-Cruz, 2007).

The question remains whether the existence of such polysemous connectives should be seen as counter-evidence to the linguistic categorization hypothesis. Given that ambiguity proliferates in language, and polysemy is not restricted to temporal connectives, we are inclined to answer this question with a no. Again, examples of temporal while that are clearly non-ambiguous can be found, and enough non-ambiguous temporal markers remain that justify recognizing temporality as a cognitively plausible feature.

\footnotetext{
${ }^{1}$ Note that this process is by no means restricted to English. For example, Dutch terwijl 'while' has, similar to while in English, taken on a contrastive meaning (Vogl, 2007), German weil 'because' also used to mark that events happened at the same time, but invited a causal inference following the pattern post hoc ergo propter hoc (Behaghel, 1928: 341; Traugott \& König, 1991: 194), and has since been semanticized, into a causal connective (Keller, 1995).
} 
In sum, given that there are specific temporal connectives that can mark temporal relations, we argue that temporal order is indeed a cognitively plausible and a relational feature. In the next sections, we will see that temporal connectives and relations are acquired and processed differently from other types of connectives.

\subsection{Evidence from language acquisition}

For temporality to be considered a cognitively plausible notion, it should play a role in the cognitive processes underlying language use. In this section, we will therefore look at evidence from language acquisition data, and in the next section, we will address processing data from adult language users. We discuss a non-exhaustive list of studies that have shown that temporality influences language use.

Language acquisition studies have shown that the first emergence of temporal connectives happens at a different stage than that of additives or causals: around their second birthday, children start combining clauses with and. Next, they start using (and) then, followed by because a few months later (see Bloom et al., 1980 for English, and Evers-Vermeul \& Sanders, 2009 for a similar developmental sequence in Dutch). The same developmental picture arises from a growth curve analysis of German connective acquisition (van Veen, Evers-Vermeul, Sanders \& van den Bergh, 2009; 2014). The fact that temporal connectives are acquired in a stage separate from both additives and causals indicates that temporal relations are a category of coherence relations that is distinct from additive and causal relations, but also that temporality is a cognitively plausible notion.

Even though children start using temporal connectives around the age of three, they are not able to fully comprehend them until much later (Bever, 1970; Blything, Davies \& Cain, 2015; Clark, 1971; French \& Brown, 1977, Johnson, 1975; Keller-Cohen, 1987; Pyykkönen, Niemi \& Järvikivi, 2003; Pyykkönen \& Järvikivi, 2012; Trosborg, 1982). In other words, children produce temporal connectives before they are able to fully comprehend them. As comprehension of temporal connectives develops slowly, there is a clearly identifiable developmental path in the process (Clark, 1971). Initially, at the age of three, children are not able to correctly interpret the temporal order information that before and after provide, using an order-of-mention strategy instead: whatever is mentioned first, is interpreted as the first event. In the next stage, around the age of four, children interpret sentences with before correctly, but not yet sentences with after. Finally, around the age of five, children are able to comprehend both temporal connectives correctly around $80 \%$ of the time.

A comprehension score of $80 \%$ is still lower than that of adults, who do not have any trouble correctly interpreting temporal order in relations (see Section 3.3). Research on when exactly children reach adult competence in interpreting these connectives does not provide a conclusive answer: for example, Blything, Davies and Cain (2015) find in a forced choice reading experiment that children are performing at high levels of accuracy around age seven, while Cain and Nash (2011) report that ten-year-olds still differed from adult levels of performance on a cloze task, sentence rating task and online reading task. Pyykkönen and Järvikivi (2012) even found that children experience difficulties achieving the correct interpretation of temporal relations containing before and after until the age of twelve. Regardless of the exact age at which children are able to successfully comprehend temporal relations, it is clear that children experience difficulty in interpreting these relations. Blything et al. (2015) attribute this difficulty to children's working memory. In a forced choice reading experiment, they show that children with higher memory capacity comprehend different types of temporal relations more accurately.

On the basis of the acquisition data discussed in this section, we can conclude that temporality is relevant for children's language production and their language comprehension from a very early age. Production data indicate that temporality determines part of the order of acquisition of connectives, and studies investigating children's comprehension of temporal relations indicate that their interpretation process is facilitated by a chronological order of events. 


\subsection{Evidence from language processing}

If temporality is a cognitively plausible notion, it should play a role in language processing in adults as well. Indeed, it has been found that readers do encode temporal information in their representations (Mandler, 1986; Gennari, 2004; Townsend, 1983; van der Meer, Beyer, Heinze \& Badel, 2002). Zwaan (1996), for example, investigated the effect of discourse time shifts such as an hour later in the context of a sequence of events (for example, John was beaming. A moment later...). Sentential reading times were longer after a large time shift such as an hour later compared to a smaller time shift such as a moment later. These results indicate that readers track the time of events while reading a text, and that the duration of the time shifts also influences the reading times. Other studies have shown that the order of events also influences readers' processing; it has consistently been found that chronologically ordered temporal relations are processed faster than anti-chronologically ordered sentences. In this section, an overview of these studies will be given.

Unlike children, adults are able to achieve the correct interpretation regardless of the event order in relations. However, chronologically ordered sentences do facilitate processing. Offline studies have shown that temporal relations with a chronological order are remembered better. For example, Clark and Clark (1968) investigated the recall of complex sentences marked by before, and then, after or but first. They found that the subjects remembered the sentences with a chronological order better than those with an anti-chronological order. Similarly, Townsend (1983) investigated the recall of temporal and causal English sentences connected by after, before, when, since, while or because and found that, for both types of relations, the subjects remembered the sentences with a chronological order better than those with an anti-chronological order. Baker (1978) found that people were faster and more accurate in reporting the order of events in narratives when the events were presented in chronological order compared to antichronological order. These studies therefore show that the temporal order of clauses affects how well the relation is encoded in the mental representation.

Münte, Schiltz and Kutas (1998) provided evidence that the encoding of temporal order by adults requires working memory as well. Their stimuli consisted of chronological and nonchronological sentences starting with either before or after, such as Before/After the psychologist submitted the article, the journal changed its policy. Using event-related potentials (ERP), they showed that the participants had different brain responses to the connectives before and after within $300 \mathrm{~ms}$ after presentation of the connective. The authors interpreted these results as indicating that anti-chronologically ordered sentences are more demanding of working memory because they require additional discourse-level computations. These findings were confirmed by Ye et al. (2012) in an fMRI study. Using stimuli similar to the ones used by Münte et al. (1998), they found that anti-chronological sentences need additional discourse-level computations to reverse the order of the two constituent clauses. Readers therefore appear to invest cognitive energy in correctly representing the temporal order of relations.

Interestingly, however, it seems that temporal relations differ from causal coherence relations in this respect. Mandler (1986) found that a chronological order of complex sentences facilitates the processing of temporal relations, but not of causal relations. In other words, chronological ordering of the relation is not necessary for rapid understanding of the temporal relation between two causally connected events. Mandler attributes this difference to the readers' world knowledge: when events are causally connected, prior knowledge about the temporal relations between a cause and its effect makes encoding the order of two events relatively effortless, regardless of the order of mention of the events. In the case of events that are arbitrarily ordered in time, more effort is required to encode the temporal order, since no pre-existing knowledge about this order is available (cf. French \& Brown, 1977; Trosborg, 1982). Hence, temporal relations seem to be processed differently from causal relations.

Taken together, the results of several offline and online experiments indicate that the temporal order of coherence relations affects the processing of these relations in adults. Although 
adults do not have the same difficulty as children with achieving the correct interpretation of temporal relations, they do process these relations faster and recall them better when the segments are ordered chronologically. An anti-chronological order of the segments requires additional computations, which is more demanding of working memory. This indicates that temporality is a cognitively relevant notion that people take into account when using language.

\section{How to implement temporality in CCR}

In Sections 2 and 3 we have shown that information on the temporal ordering of segments is relational information and that temporality is a cognitively plausible notion; temporal order is expressed in the system of linguistic markers, and is relevant in acquisition as well as language processing. This entails that temporality should be implemented in CCR. For many other approaches, it would be fairly easy to extend the respective inventory of relation labels by adding more labels. However, CCR does not work with an inventory of relation labels; rather, it distinguishes a restricted set of four dimensions that are relevant for describing all types of relations (Sanders et al., 1992, 1993). As a result, including temporality in the framework is not as straightforward as it might be in other discourse annotation frameworks. In the remainder of this section we will examine two options of including temporality in CCR: to extend one of the existing CCR dimensions in order to incorporate temporality (Section 4.1), and adding a new dimension to CCR (Section 4.2). First we will briefly introduce the relevant CCR dimensions.

\subsection{Extending a current CCR dimension}

CCR differs from other annotation frameworks in that coherence relations are not assigned a specific end label, but rather are defined by their characteristics. In CCR, four cognitive dimensions are distinguished that apply to every relation. These dimensions are polarity, source of coherence, basic operation, and order of the segments (Sanders et al., 1992). Here we explore whether one or both of the latter two dimensions can be adapted in order to include temporal relations in CCR.

Basic operation distinguishes between causal and additive relations. A relation is causal if an implication relation $(\mathrm{P} \rightarrow \mathrm{Q})$ can be deduced between the two segments. Causal relations are typically connected by because or so. A relation is additive if the relation between the segments is one of logical conjunction ( $\mathrm{P} \& \mathrm{Q})$. Additive relations are typically connected by and. In the original proposal, CCR considers temporal relations to be a subtype of additive relations.

One way of giving temporality a separate status in CCR would be to add the value 'temporal' to the basic operation dimension, thereby making a three-way distinction between additive, temporal, and causal relations. This option has actually already been put into practice by, for example, Louwerse (2001) and Scholman, Evers-Vermeul and Sanders (2016). However, this option ignores the fact that other CCR dimensions are binary, distinguishing, for instance, positive vs. negative relations, and basic vs. non-basic relations. ${ }^{2,3}$

\footnotetext{
${ }^{2}$ Note, however, that the principle of having binary dimensions has been 'violated' before. On the dimension source of coherence, sometimes two values are distinguished: semantic vs. pragmatic (Sanders et al., 1992), or objective vs. subjective (Scholman et al., 2016), but it is also frequently operationalized as having three values, following Sweetser's (1990) approach: content, epistemic and speech act (see, among others, Evers-Vermeul, 2005; Stukker, 2005). This trichotomy, however, is really a simplification of two binary distinctions: objective (content) vs. subjective, which contains both epistemic and speech act relations. This allows for different kinds of generalizations: first, between content on the one hand and epistemic and speech act on the other, and second between epistemic and speech act.

${ }^{3}$ In line with other cognitive linguists, we acknowledge the fact that in practice categories often display fuzzy boundaries, with less and more prototypical instances (cf. Rosch, 1977). This has also been explored for coherence relations and connectives (Stukker \& Sanders, 2012; Sanders \& Spooren, 2013). This does not, however, mean that the definitions of the categories themselves should be fuzzy. Given the objectives of the current paper, we will not address the fundamental issue of whether we should abandon binary distinctions in favor of the prototypes typically used in cognitive linguistics, but instead base our line of reasoning on the binary categorizations that have shown to be relevant in accounting for patterns in both language acquisition and language use.
} 
More importantly, it ignores the fact that many causal relations display a temporal order as well; by default, causes in the real world precede their consequence. By placing temporal order on a par with causality, the two values become mutually exclusive. Note, however, that all causal relations are by default additive as well, given that an implication relation $(\mathrm{P} \rightarrow \mathrm{Q})$ presupposes $\mathrm{P}$ and Q (P \& Q). In other words, this overlap is inevitable. An important difference between causal vs. additive relations and causal vs. temporal relations is that not all causal relations are temporal, and that during the annotation process one would therefore want to be able to attribute these values simultaneously in order to be able to distinguish between temporal causal relations and non-temporal causal relations. We will further explore this point in Section 4.2.

A second option would be to adapt the CCR dimension order of the segments, as is done in Scholman et al. (2016). In the original CCR proposal (Sanders et al., 1992), order of the segments is defined in terms of implication relations, and therefore only applies to causal and conditional relations. It refers to the mapping of the antecedent $(\mathrm{P})$ and the consequent $(\mathrm{Q})$ onto the segments, where $\mathrm{P}$ is the cause, condition or argument, and $\mathrm{Q}$ is the consequence or the claim. In a coherence relation with a basic order, such as (20), $\mathrm{P}$ is $\mathrm{S}_{1}$, followed by $\mathrm{Q}$ as $\mathrm{S}_{2}$. In a relation with a non-basic order, such as (21), P maps onto $\mathrm{S}_{2}$ and $\mathrm{Q}$ onto $\mathrm{S}_{1}$.

(20) [It was raining.] $\mathrm{S}_{1}$ That is why [the streets are wet.] $\mathrm{S}_{2}$

(21) [The streets are wet] $\mathrm{S}_{1}$ because [it was raining. $] \mathrm{S}_{2}$

In the original proposal, the order of the segments dimension does not apply to additive relations, as these are symmetrical by definition. However, it could be argued that segments with a sequential temporal order form an exception in this respect because the events expressed in $\mathrm{P}$ and $\mathrm{Q}$ are necessarily ordered in time. Following this line of reasoning, it could be said that if $\mathrm{S}_{1}$ and $\mathrm{S}_{2}$ follow this order in time, the relations show a basic order. In a relation with a non-basic order, $\mathrm{S}_{2}$ expresses $\mathrm{P}$; that is, the event expressed in $\mathrm{S}_{2}$ precedes the event in $\mathrm{S}_{1}$ in reality.

There are some drawbacks to depicting the chronological ordering of temporal relations using order of the segments. First and foremost, it would require seriously stretching or altering the definition of order of the segments. Temporal relations, after all, do not involve an implication relation and do not consist of an antecedent $\mathrm{P}$ and a consequent $\mathrm{Q}$.

Second, for causal and conditional relations, this solution does not leave the option of distinguishing between the order of the segments within the implication relation and the chronological order of the segments. As we will elaborate on in Section 4.2, these two types of order may, but do not necessarily overlap.

Third, if the values on the basic operation dimension are still restricted to additive and causal, as in the original CCR proposal, this solution implies that in principle, order of the segments becomes available for the entire class of additives. In this scenario, temporal relations can be distinguished from other additive relations by looking at their value for order, and additive relations that are not specified for order would be purely additive relations. However, CCR would then have no way of setting apart purely additive relations from temporal relations expressing temporal overlap. Since both additive and synchronous temporal relations are symmetrical in the sense that neither relation involves one segment occurring before the other, this would mean that neither type would be specified for order.

Considering the initial motivations of Sanders et al.'s (1992) taxonomy of coherence relations and considering the drawbacks mentioned above, using order of the segments to capture the chronological ordering of temporal relations seems undesirable.

\subsection{Adding a new dimension to CCR}

Considering the drawbacks of using one of the existing dimensions to incorporate temporal relations in CCR, as outlined in Section 4.1, the only option for achieving this goal seems to be adding one or more new dimensions to CCR's inventory of annotation dimensions. Adding one 
extra dimension would be in line with Evers-Vermeul and Sanders (2009), who distinguish between [+ temporal] and [ $\alpha$ temporal] relations in order to be able to account for the order of first emergence of the English connectives and, then, and because, and their Dutch counterparts. Adding more than one temporality dimension would be in line with Clark (1971: 273), who presents three hierarchically ordered features to account for the acquisition of the temporal connectives after, before and when: [+/- Time], [+/-Simultaneous], and [+/- Prior]. According to Clark, children's production data reflect the learning of these features from the most superordinate feature [+ Time] down, which reflects the cognitive relevance of all three features.

Below, we will take this latter approach, and propose to implement the three-step temporality dimension presented in (22) that is orthogonal to the other CCR dimensions. This dimension will help account for temporal relations within CCR while adhering to its initial basic principle of using binary variables. Note that this approach of working with a dimension with multiple, hierarchically ordered steps can already be found in CCR itself, even though it is not presented as such. First, CCR applies the basic operation dimension to set implication relations apart from additive relations. Second, within the group of implication relations, causal relations are distinguished from conditional relations. Third, Sanders et al. (1992: 12) propose that volitionality would be a candidate for further specification of the proposed taxonomy, which would then differentiate volitional and non-volitional causal relations.

(22) The three-step temporality dimension

\begin{tabular}{|c|c|c|c|c|}
\hline 1 & \multicolumn{3}{|l|}{ Temporal } & Non-temporal \\
\hline 2 & \multicolumn{2}{|l|}{ Sequential } & Synchronous & \\
\hline 3 & Chronological & Anti-chronological & & \\
\hline
\end{tabular}

As we have mentioned before, CCR considers temporal relations to be a subset of additive relations; that is, temporal relations are considered to be additive relations that are ordered in time. The first step in the temporality dimension we propose helps distinguish the subset of temporal relations from the rest of the additive relations. Relations such as (23), (24) and (25) are temporal, while additive relations such as (26) are non-temporal. ${ }^{4}$

(23) After [Mark came home from the supermarket, $] \mathrm{S}_{1}[$ he realized his grocery list continued on the back of the paper.] $\mathrm{S}_{2}$

(24) Before [Anne went to the important meeting, $] \mathrm{S}_{1}$ [she did a lot of preparation.] $\mathrm{S}_{2}$

(25) [Mirjam played the piano,] $\mathrm{S}_{1}$ while [Nathan was playing soccer.] $\mathrm{S}_{2}$

(26) [Michael works at the veterinary clinic across the street. $] \mathrm{S}_{1}[\mathrm{He}$ also volunteers at the local animal shelter.] $\mathrm{S}_{2}$

To the additive relation in (26), chronological ordering is irrelevant. Although in terms of truthvalue $S_{1}$ and $S_{2}$ hold simultaneously, these segments are not presented in combination to indicate that Michael performs these activities simultaneously in the real world. Note that temporal only means that temporality is relevant to the relation at hand, not necessarily that the segments display a sequence of events. Hence, the synchronous temporal relation in (25) and the sequential relations in (23) and (24) are all temporal.

\footnotetext{
${ }^{4}$ We leave it to another paper to discuss whether values distinguished within a dimension should simply receive different value labels, or whether these would have to represented with actual features such as [+/- Time] or [+/ $\alpha$ Time]. In line with the labeling within other CCR dimensions, we take the first approach, and use different value labels.
} 
The second step in the temporality dimension distinguishes between sequential and synchronous temporal relations. Since in (23) and (24) one of the segments occurs before the other, these temporal relations are sequential. By contrast, the non-sequential relation in (25), in which the segments display temporal overlap, is labeled synchronous.

Finally, the chronological order of sequential temporal relations has to be determined. This distinction is captured by the third step in the temporality dimension, which differentiates between chronological and anti-chronological relations. Since the event in $\mathrm{S}_{1}$ occurs before the event in $\mathrm{S}_{2},(23)$ has a chronological order. In (24), the order of the segments does not match the order of the events in the real world. This relation is therefore anti-chronological.

Because the temporality dimension operates orthogonal to the other CCR dimensions, the three steps do not only apply to additive relations. They are also relevant to objective causal relations, such as (27) and (28), which depict an implication relation that can be observed in the real world. Temporal ordering is involved in both cases, which is why they are both labeled temporal. In addition, both relations are sequential, as the events follow each other in time. Finally, (27) is chronological because the freezing mentioned in $\mathrm{S}_{1}$ occurs first and causes the formation of the ice flowers mentioned in $\mathrm{S}_{2}$. By contrast, (28) is anti-chronological because the freezing is mentioned in $\mathrm{S}_{2}$.

(27) [It had been freezing.] $\mathrm{S}_{1}$ That is why [there were ice flowers on the windows.] $\mathrm{S}_{2}$

(28) [There were ice flowers on the windows] $\mathrm{S}_{1}$ because [it had been freezing.] $\mathrm{S}_{2}$

In these examples, the values on the third step in the temporality dimension align with the values on the dimension order of the segments: (27) is chronological and displays a basic order, since $\mathrm{S}_{1}$ expresses the antecedent $\mathrm{P}$ and $\mathrm{S}_{2}$ the consequent $\mathrm{Q}$, while (28) is anti-chronological and has a non-basic order. Chronological ordering and order of the segments will align in this way, basic/chronological and non-basic/anti-chronological, in all objective causal relations, as a cause tends to precede its effect.

However, chronological ordering can, and often will, differ from the order of the segments in case of subjective causal relations, which includes both epistemic and speech act relations (Sanders \& Spooren, 2009). Consider for instance the relations in (29) and (30).

(29) [The neighbors' lights are on, $] \mathrm{S}_{1}$ so [they must be home.] $\mathrm{S}_{2}$

(30) [The neighbors just got home, $] \mathrm{S}_{1}$ so [the lights will probably be turned on soon.] $\mathrm{S}_{2}$

Both (29) and (30) have a basic order of the segments: $\mathrm{P}$ is expressed in $\mathrm{S}_{1}$, while $\mathrm{S}_{2}$ expresses Q, which in both cases is a judgment rather than a fact. The temporal order of events in the real world, however, is not identical. (29) is ordered anti-chronologically, since turning on the lights, depicted in $\mathrm{S}_{1}$, happens after the neighbors' coming home, which is depicted in $\mathrm{S}_{2}$. (30), on the other hand, is ordered chronologically: the neighbors' arrival in $\mathrm{S}_{1}$ precedes the probable turning on the lights in $\mathrm{S}_{2}$. By using the third step in the temporality dimension in addition to order of the segments, we can capture the difference between relations in which an argument is presented to back up a claim or conclusion, as in (29), and relations in which a prediction is made on the basis of an observation, as in (30).

Distinguishing between chronological order and order of the segments helps analyze not only subjective causal relations, but also relations such as (31), also known as IMPLICIT ASSERTION relations in the PDTB (PDTB Research Group, 2008).

(31) If [you want to become rich someday,] $\mathrm{S}_{1}$ [you should probably get off the couch.] $\mathrm{S}_{2}$

(31) is a subjective conditional relation that has a basic order of the segments: the antecedent $\mathrm{P}$ is expressed in $\mathrm{S}_{1}$, and the consequent $\mathrm{Q}$ in $\mathrm{S}_{2}$. However, in real time, the getting off the couch in $\mathrm{S}_{2}$ 
would have to occur before the getting rich in $\mathrm{S}_{1}$. (31) therefore has an anti-chronological temporal order. Other examples of relations that could be annotated along these lines would be RST-DT's ENABLEMENT and PROBLEM-SOLUTION.

In sum, we have proposed to incorporate temporality into CCR by implementing a temporality dimension with three hierarchically ordered binary annotation steps that are orthogonal to other CCR dimensions such as basic operation and order of the segments.

\section{Discussion and conclusion}

In this paper, we have argued that information on the temporal ordering of segments in a discourse cannot be seen as merely segment-specific information, and that temporal order should thus be considered a relational feature, even in the presence of linguistic indicators of temporality within the connected clauses. The Cognitive approach to Coherence Relations considers coherence relations to be cognitive entities. This means that if temporal relations are to be included in CCR, the defining features that separate temporal relations from other types of relations should be cognitively plausible. A review of empirical evidence from the system of linguistic markers, from language acquisition, and from language processing supports the claim that temporal features are indeed cognitively plausible.

In Section 4, we have therefore presented a proposal on how to implement temporality in CCR. We suggest adding a three-step temporality dimension to CCR with a hierarchically ordered set of binary annotation steps that are orthogonal to the other dimensions, including basic operation and order of the segments. The first step in the temporality dimension sets temporal relations apart from non-temporal relations, in which temporal order is not relevant. The second step in the temporality dimension distinguishes between sequential and synchronous relations, and the third step differentiates between chronological and anti-chronological relations.

The proposed approach allows for generalizing over different subtypes of temporal relations. For example, relations that are classified as additive on basic operation and temporal on the first temporality dimension, constitute the class of TEMPORALS distinguished by for instance PDTB and RST-DT (see Section 1). In addition, the new temporality dimensions allow for a different type of generalization, one that none of the current discourse annotation frameworks are able to make. On top of setting apart relations for which temporality is the key defining feature, it can cluster all relations for which temporality is a relevant feature. This allows us to show the similarities between sequential temporal relations and objective causal relations, and between synchronous temporal relations and contrastive relations. It is exactly this type of clustering we need in order to be able to account for frequent linguistic phenomena such as underspecification and pragmatic enrichment (see Section 3.1). In addition, the temporal dimension can help distinguish between different types of subjective causal relations and make explicit that in subjective causal relations temporal order may not always align with order of the segments (see Section 4.2).

We have drawn a clear distinction between temporality at the sentence level (the location of events on a time line as indicated by past, present or future tense, and the temporal constituency of events as expressed by markers of aspect) and temporality at the discourse level, focusing on the relational surplus of temporal order. This approach differs from the one taken by researchers who seem to collapse these levels and, for example, label 'discourse verbs' (Danlos, 2006; Lejeune, Mendes \& Martins, 2016) and prepositional phrases taking nominal arguments (Atallah, Bras \& Vieu, 2015; Mendes \& Lejeune, 2016; Rysová \& Rysová, 2015; Stede, 2002) as markers of coherence relations. Although we acknowledge the merit of studying such alternative lexicalizations (cf. Prasad et al., 2010), we prefer not to categorize alternative lexicalizations as markers of coherence relations, that is, as markers of the relational surplus of coherence relations. Keeping the discourse and the sentence levels apart allows us to investigate how markers of 
temporality - or, for instance, causality - at these two levels interact. This may, for example, generate insight into why certain combinations of sentence and discourse markers are possible, preferred or impossible. For example, Knott and Dale (1994: 50) observe that earlier, afterwards, and later, as well as before and after can all be modified by any expression denoting a length of time, for instance, three days after, a minute earlier, and some time before. By contrast, the combination of certain temporal connectives and 'discourse verbs' denoting progression of time is impossible, as the comparison of (32), (33) and (34) illustrates (example (34) taken from Danlos, 2006: 65).

(32) Ted left. Next, Sue arrived.

(33) Ted left. This preceded Sue's arrival.

(34) \#Ted left. Next, this preceded Sue's arrival.

From a cognitive perspective, studying similarities and differences between marking at the sentence and the discourse level may give us insight into the underlying cognitive categories that people make use of. For instance, Stukker, Sanders and Verhagen (2008) investigate causality at the inter-clausal level (expressed by causal connectives) and at the intra-clausal level (expressed by causal verbs), which allows them to show commonalities between the two. From a developmental perspective, linguists can and already do study the way in which markers that have a function at the sentence level gradually obtain a function at the discourse level. A famous example is the development of while discussed by Traugott (1995), but more generally, one could look at differences between so-called primary and secondary, less grammaticalized connectives (Rysová \& Rysová, 2015). This would be in line with Knott and Dale's (1994:49-50) observation that relational markers are sometimes single elements and idiom chunks (e.g. because, on the other hand), but can also be partly compositional (five minutes later, three years later). Also, researchers can account for the way in which children over the years acquire the various grammatical, lexical and discourse devices for expressing temporality (Berman \& Slobin, 1994; Uccelli, 2009).

In this paper, we have focused on the question whether temporality should be considered a relational or a segment-specific feature. The same question can be raised for other types of relations for which annotators need to decide if they should distinguish these by adding a separate relation label to their relation inventory. Similarly, the method used in this paper could be used to decide whether a relation label should be maintained within an inventory of coherence relations, or whether the label is not necessary because there is no relational surplus and further specifications can be made on the basis of segment-specific features. In their comparison of annotation frameworks, Sanders et al. (submitted) have listed several features that are distinguished in one or more of the other frameworks but not in CCR, thereby presenting a list of candidates for which this discussion seems relevant. This includes SPECIFICITY, which seems to play a role in INSTANTIATION relations and the subtypes of RESTATEMENTS in PDTB, as well as in RST-DT's collection of ELABORATION RELATIONS. Other candidates that require further investigation are LIST, which sets apart LIST relations from other types of additive relations, and features that discriminate between the different types of CONDITIONALS found in PDTB and RST-DT. We have shown that the cognitive plausibility of the relation type under investigation can be tested fruitfully using empirical evidence from acquisition data, processing, and the system of linguistic markers.

\section{Acknowledgements}

The first author's work was enabled by a grant awarded by the Executive Board of Utrecht University to the AnnCor project, work package Discourse Annotation. The second author was funded by the SNSF Sinergia project MODERN (CRSII2_147653). The third author was funded 
by the German Research Foundation (DFG) as part of SFB 1102 "Information Density and Linguistic Encoding”.

\section{References}

Barbara E. Adam (2008). The timescapes challenge: Engagement with the invisible temporal. In B. E. Adam, J. Hockey, P. Thompson, Paul and R. Edwards (eds.), Researching Lives Through Time: Time, Generation and Life Stories, Timescapes Working Paper Series, Volume 1: 7-12. Leeds: University of Leeds.

Nicholas Asher and Alex Lascarides (2003). Logics of Conversation. Cambridge: Cambridge University Press.

Fatemeh T. Asr and Vera Demberg (2015). Uniform information density at the level of discourse relations: Negation markers and discourse connective omission. In IWCS 2015, pages 118 128, London, United Kingdom.

Caroline Atallah, Myriam Bras and Laure Vieu (2015). Discourse relations, discourse connectives and discourse segmentation interdependency in the light of causality. Paper presented at LPTS2016. Valencia, Spain.

Linda Baker (1978). Processing temporal relationships in simple stories: Effects of input sequence. Journal of Verbal Learning and Verbal Behavior, 17(5): 559-572.

Michael Barlow and Suzanne Kemmer (2000). Usage Based Models of Language. Stanford: CSLI Publications.

Otto Behaghel (1928). Deutsche Syntax. Die Satzgebilde, Band III. Heidelberg: Carl Winters Universitätsbuchhandlung.Ruth Berman and Dan I. Slobin (1994). Relating Narrative Events: A Crosslinguistic Developmental Study. Hillsdale, NJ: Lawrence Erlbaum.

Yves Bestgen and Jean Costermans (1994). Time, space, and action: Exploring the narrative structure and its linguistic marking. Discourse Processes, 17(3): 421-446.

Thomas G. Bever (1970). The cognitive basis for linguistic structures. In R. Hayes (ed.), Cognition and Language Development: 279-362. New York: Wiley \& Sons.

Diane Blakemore (2002). Relevance and Linguistic Meaning: The Semantics and Pragmatics of Discourse Markers. Cambridge: Cambridge University Press.

Lois Bloom, Margaret Lahey, Lois Hood, Karin Lifter and Kathleen Fiess (1980). Complex sentences: Acquisition of syntactic connectives and the semantic relations they encode. Journal of Child Language, 7(2): 235-261.

Liam P. Blything, Robert L. Davies and Kate E. Cain (2015). Young children's comprehension of temporal relations in complex sentences: The influence of memory on performance. Child Development, 86(6): 1922-1934.

Bruce K. Britton (1994). Understanding expository text: Building mental structures to induce insights. In M. A. Gernsbacher (ed.), Handbook of Psycholinguistics: 641-674. San Diego, California: Academic Press.

Harry Bunt and Rashmi Prasad (2016). ISO DR-Core (ISO 24617-8): Core Concepts for the Annotation of Discourse Relations. In Proceedings 12th Joint ACL-ISO Workshop on Interoperable Semantic Annotation (ISA-12), pages 45-54, Portoroz, Slovenia.

Kate E. Cain and Hannah M. Nash (2011). The influence of connectives on young readers' processing and comprehension of text. Journal of Educational Psychology, 103(2): 429-441.

Anneloes R. Canestrelli, Willem M. Mak and Ted J.M. Sanders (2013). Causal connectives in discourse processing: How differences in subjectivity are reflected in eye-movements. Language and Cognitive Processes, 28(9): 1394-1413.

Lynn Carlson, Daniel Marcu and Mary E. Okurowski (2003). Building a discourse-tagged corpus in the framework of Rhetorical Structure Theory. In J. van Kuppevelt and R. Smith (eds.), Current Directions in Discourse and Dialogue: 85-112. Dordrecht: Kluwer Academic Publishers. 
Eve V. Clark (1971). On the acquisition of the meaning of before and after. Journal of Verbal Learning and Verbal Behavior, 10(3): 266-275.

Herbert H. Clark and Eve V. Clark (1968). Semantic distinctions and memory for complex sentences. The Quarterly Journal of Experimental Psychology, 20(2): 129-138.

Bernard Comrie (1976). Aspect. Cambridge: Cambridge University Press

Jean Costermans and Yves Bestgen (1991). The role of temporal markers in the segmentation of narrative discourse. CPC: European Bulletin of Cognitive Psychology, 11, 349-370.

Laurence Danlos (2006). 'Discourse verbs' and discourse periphrastic links. In C. Sidner, J. Harpur, A. Benz and P. Kühnlein (eds.), Proceedings of the Second Workshop on Constraints in Discourse (pp.59-65). Maynooth, Ireland.

Evers-Vermeul, J. (2005). The development of Dutch connectives: Change and acquisition as windows on form-function relations. $\mathrm{PhD}$ thesis, Utrecht University. Utrecht: LOT. Available at: http://www.lotpublications.nl/Documents/110_fulltext.pdf.

Jacqueline Evers-Vermeul and Ted J.M. Sanders (2009). The emergence of Dutch connectives: how cumulative cognitive complexity explains the order of acquisition. Journal of Child Language, 36(4): 829-854.

Lucia A. French and Ann L. Brown (1977). Comprehension of before and after in logical and arbitrary sequences. Journal of Child Language, 4(2): 247-256.

Silvia P. Gennari (2004). Temporal references and temporal relations in sentence comprehension. Journal of Experimental Psychology: Learning, Memory, and Cognition, 30(4): 877-890.

Talmy Givón (1990). Syntax: A Functional-Typological Introduction, Volume 2. Amsterdam/ Philadelphia: Benjamins.

Ana I. González-Cruz (2007). On the subjectification of adverbial clause connectives: Semantic and pragmatic considerations in the development of while-clauses. In U. Lenker and A. Meurman-Solin (eds.), Connectives in the History of English: 145-166. Amsterdam/ Philadelphia: John Benjamins.

H. Paul Grice (1989). Studies in the Way of Words. Cambridge, MA/London: Harvard University Press.

Michael A. K. Halliday and Ruqaiya Hasan (1976). Cohesion in English. London: Longman.

Alison Hall (2007). Do discourse connectives encode concepts or procedures? Lingua, 117(1): 149-174.

Erhard Hinrichs (1986). Temporal anaphora in discourses of English. Linguistics and Philosophy, 9: 63-82.

Jerry R. Hobbs (1983). Why is discourse coherent? In F. Neubauer (ed.), Coherence in Natural Language Texts: 29-70. Hamburg: Buske.

Eduard H. Hovy and Elisabeth Maier (1995). Parsimonious or profligate: How many and which discourse structure relations. Unpublished manuscript. Available at: http://www.isi.edu/natural-language/people/hovy/papers/93discproc.pdf.

Helen L. Johnson (1975). The meaning of before and after for preschool children. Journal of Experimental Child Psychology, 19(1): 88-99.

Andrew Kehler (1994). Temporal relations: Reference or discourse coherence? In Proceedings of the 32nd Annual Conference of the Association for Computational Linguistics (ACL-94), pages 319-321, Las Cruces, New Mexico.

Andrew Kehler (2002). Coherence, Reference, and the Theory of Grammar. Stanford: CSLI Publications.

Rudi Keller (1995). The epistemic weil. In D. Stein and S. Wright (eds.), Subjectivity and Subjectivisation: Linguistic Perspectives: 16-30. Cambridge: Cambridge University Press.

Deborah Keller-Cohen (1987). Context and strategy in acquiring temporal connectives. Journal of Psycholinguistic Research, 16(2): 165-183.

Alistair Knott and Robert Dale (1994). Using linguistic phenomena to motivate a set of coherence relations. Discourse Processes, 18: 35-62. 
Alex Lascarides and Nicolas Asher (1993). Temporal interpretations, discourse relations and common sense entailment. Linguistics and Philosophy, 16(5): 437-493.

Pierre Lejeune, Amália Mendes and Nuno Martins (2016). Some considerations on the use of main verbs to express rhetorical relations. In L. Degand, C. Dér, P. Furkó, and B. Webber (eds.). Conference Handbook TextLink - Structuring Discourse in Multilingual Europe Second Action Conference (pp.81-85). Budapest: Debrecen University Press.

Stephen C. Levinson (2000). Presumptive Meaning: The Theory of Generalized Conversational Implicature. Cambridge, MA/London: MIT Press.

Robert E. Longacre (1983). The Grammar of Discourse: Notional and Surface Structures. Plenum: New York.

Max Louwerse (2001). An analytic and cognitive parameterization of coherence relations. Cognitive Linguistics, 12(3): 291-315.

Willem M. Mak and Ted J.M. Sanders (2013). The role of causality in discourse processing: Effects of Expectation and Coherence relations. Language and Cognitive Processes, 28: 14141437.

Jean M. Mandler (1986). On the comprehension of temporal order. Language and Cognitive Processes, 1(4):309-320.

James R. Martin (1992). English Text: System and Structure. Amsterdam/Philadelphia: John Benjamins.

Amália Mendes and Pierre Lejeune (2016). LDM-PT: A Portuguese lexicon of disourse markers. In L. Degand, C. Dér, P. Furkó, and B. Webber (eds.). Conference Handbook TextLink Structuring Discourse in Multilingual Europe Second Action Conference (pp.89-92). Budapest: Debrecen University Press.

Thomas F. Münte, Kolja Schiltz and Marta Kutas (1998). When temporal terms belie conceptual order. Nature, 395: 71-73.

John Nerbonne (1986). Reference time and time in narration. Linguistics and Philosophy, 9: 8395.

Barbara H. Partee (1984). Nominal and temporal anaphora. Linguistics and Philosophy, 7: 243286.

PDTB Research Group (2008). The Penn Discourse Treebank 2.0 Annotation Manual. Technical Report IRCS-08-01. Philadelphia: Institute for Research in Cognitive Science, University of Pennsylvania. Available at: https://www.seas.upenn.edu/ pdtb/PDTBAPI/pdtb-annotationmanual.pdf.

Rashmi Prasad, Nikhil Dinesh, Alan Lee, Eleni Miltsakaki, Livio Robaldo, Aravind K. Joshi and Bonnie L. Webber (2008). The Penn Discourse Treebank 2.0. In Proceedings of the $6^{\text {th }}$ International Conference of Language Resources and Evaluation (LREC 2008), Marrakech, Morocco. Available at: https://www.seas.upenn.edu/ pdtb/papers/pdtb-lrec08.pdf.

Rashmi Prasad, Aravind K. Joshi and Bonnie L. Webber (2010). Realization of discourse relations by other means: Alternative lexicalizations. In Proceedings of the 23rd International Conference on Computational Linguistics (COLING 2010), Posters Volume, pages 10231031, Beijing, China.

Pirita Pyykkönen and Juhani Järvikivi (2012). Children and situation models of multiple events. Developmental Psychology, 48(2): 521-529.

Pirita Pyykkönen, Jussi Niemi and Juhani Järvikivi (2003). Sentence structure, temporal order and linearity: Slow emergence of adult-like syntactic performance in Finnish. SKY Journal of Linguistics, 16: 113-138.

Brian J. Reese, Julie Hunter, Nicholas Asher, Pascal Denis and Jason Baldridge (2007). Reference manual for the analysis of rhetorical structure. Technical Report, University of Texas at Austin, Austin, Texas. 
Eleanor Rosch (1977). Classification of real-world objects: Origins and representations in cognition. In P.N. Johnson-Laird \& P.C. Wason (eds.), Thinking: Readings in Cognitive Science (pp.212-222). Cambridge: Cambridge University Press.

Magdaléna Rysová (2012). Alternative lexicalizations of discourse connectives in Czech. In Proceedings of the Eight International Conference on Language Resources and Evaluation (LREC'12), pages 2800-2807, Istanbul, Turkey.

Magdaléna Rysová and Kateřina Rysová (2015). Secondary connectives in the Prague Dependency Treebank. In Proceedings of the Third International Conference on Dependency Linguistics (Depling 2015), pages 291-299, Uppsala. Sweden.

Ted J.M. Sanders, Vera Demberg, Jet Hoek, Merel C.J. Scholman, Fatemeh Torabi Asr, Sandrine Zufferey and Jacqueline Evers-Vermeul (submitted). Unifying dimensions in coherence relations: How various annotation frameworks are related. Submitted for publication.

Ted J.M. Sanders and Leo G.M. Noordman (2000). The role of coherence relations and their linguistic markers in text processing. Discourse Processes, 29: 37-60.

Ted Sanders and Wilbert Spooren (2009). Causal categories in discourse: Converging evidence from language use. In T. Sanders and E. Sweetser (eds.), Causal Categories in Discourse and Cognition (pp. 205-246). Berlin: Mouton de Gruyter.

Ted Sanders and Wilbert Spooren (2013). Exceptions to rules: A qualitative analysis of backward causal connectives in Dutch naturalistic discourse. Text \& Talk, 33(3): 377-398.

Ted J.M. Sanders, Wilbert P.M. Spooren and Leo G.M. Noordman (1992). Toward a taxonomy of coherence relations. Discourse Processes, 15: 1-35.

Ted J.M. Sanders, Wilbert P.M. Spooren and Leo G.M. Noordman (1993). Coherence relations in a cognitive theory of discourse representation. Cognitive Linguistics, 4(2): 93-133.

Joanne Scheibman (2002). Point of View and Grammar: Structural Patterns of Subjectivity in American English Conversation. Amsterdam: John Benjamins

Merel C.J. Scholman, Jacqueline Evers-Vermeul and \& Ted J.M. Sanders (2016). Categories of coherence relations in discourse annotation: Towards a reliable categorization of coherence relations. Dialogue and Discourse, 7(2): 1-28.

Wilbert Spooren (1997). The processing of underspecified coherence relations. Discourse Processes, 24: 149-168.

Manfred Stede (2002). DiMLex: A lexical approach to discourse markers. In A. Lenci and V. Di Tomaso (eds.), Exploring the Lexicon: Theory and Computation (pp.1-15). Alessandria: Edizioni dell'Orso.

Ninke Stukker (2005). Causality marking across levels of language structure. A cognitive semantic analysis of causal verbs and causal connectives in Dutch. $\mathrm{PhD}$ thesis, Utrecht University. Utrecht: LOT. Available at: http://www.lotpublications.nl/Documents/ 118 fulltext.pdf.

Ninke M. Stukker and Ted J.M. Sanders (2012). Subjectivity and prototype structure in causal connectives: A cross-linguistic perspective. Journal of Pragmatics, 44: 169-190.

Ninke Stukker, Ted Sanders and Arie Verhagen (2008). Causality in verbs and in discourse connectives: Converging evidence of cross-level parallels in Dutch linguistic categorization. Journal of Pragmatics, 40: 1296-1322.

Elizabeth C. Traugott (1995). Subjectification in grammaticalisation. In D. Stein and S. Wright (eds.), Subjectivity and Subjectivisation: Linguistic Perspectives: 31-54. Cambridge: Cambridge University Press.

Elizabeth C. Traugott (2010). (Inter)subjectivity and (inter)subjectification: a reassessment. In K. Davidse, L. Vandelanotte and H. Cuyckens (eds.), Subjectification, Intersubjectification and Grammaticalization: 29-71. Berlin \& New York: de Gruyter Mouton.

Elizabeth, C. Traugott (2011). Grammaticalization and mechanisms of change. In H. Narrog and B. Heine (eds.), The Oxford Handbook of Grammaticalization: 19-30. Oxford: Oxford University Press. 
Elizabeth C. Traugott and Ekkehard König (1991). The semantics-pragmatics of grammaticalization revisited. In E.C. Traugott and B. Heine (eds.), Approaches to Grammaticalization, Volume I: 189-218. Amsterdam/Philadelphia: Benjamins.

Anna Trosborg (1982). Children's comprehension of 'before' and 'after' reinvestigated. Journal of Child Language, 9(2): 381-402.

David J. Townsend (1983). Thematic processing in sentences and texts. Cognition, 13(2): 223261.

Paola Uccelli (2009). Emerging temporality: Past tense and temporal/aspectual markers in Spanish-speaking children's intraconversational narratives. Journal of Child Language, 36(5): 929-966.

Elke van der Meer, Reinhard Beyer, Bertram Heinze and Isolde Badel (2002). Temporal order relations in language comprehension. Journal of Experimental Psychology: Learning, Memory, and Cognition, 28(4): 770-779.

Rosie van Veen, Jacqueline Evers-Vermeul, Ted Sanders and Huub van den Bergh (2009). Parental input and connective acquisition: A growth curve analysis. First Language, 29(3): 267-289.

Rosie van Veen, Jacqueline Evers-Vermeul, Ted Sanders and Huub van den Bergh (2014). "Why? Because I'm talking to you!" Parental input and cognitive complexity as determinants of children's connective acquisition. In H. Gruber \& G. Redeker (eds.), The Pragmatics of Discourse Coherence: Theories and Applications: 209-242. Amsterdam/Philadelphia: John Benjamins.

Ulrike Vogl (2007). Het belang van conditionaliteit voor de ontwikkeling van temporeel naar causaal voegwoord: De geschiedenis van dewijl, terwijl, weil en while [The importance of conditionality in the development from temporal to causal complementizer: The history of dewijl, terwijl, weil and while]. Nederlandse Taalkunde, 12(1): 2-24.

Bonnie L. Webber (1987). The interpretation of tense in discourse. In Proceedings of the 25th annual meeting on Association for Computational Linguistics, pages 147-154, Stroudsburg, Pennsylvania.

Bonnie L. Webber (1988). Tense as discourse anaphor. Computational Linguistics, 14(2): 61-73.

Zheng Ye, Marta Kutas, Marie St George, Martin I. Sereno, Feng Ling and Thomas F. Münte (2012). Rearranging the world: Neural network supporting the processing of temporal connectives. NeuroImage, 59(4): 3662-3667.

Rolf A. Zwaan, (1996). Processing narrative time shifts. Journal of Experimental Psychology: Learning, Memory, and Cognition, 22(5): 1196-1207. 